\title{
L'approche éthique d'ordre autorégulatoire en milieu professionnel : la place de l'allégorie comme source de motivation à participer au changement
}

\author{
Marc Jean \\ Université du Québec à Chicoutimi
}

Un questionnement éthique est actuellement présent dans l'ensemble de la société, voilà un fait sociologique devenu incontournable de nos jours. Ce questionnement fait l'objet de nombreuses revendications tant au plan culturel que politique et économique. Comment favoriser l'insertion de questions éthiques telles que l'avortement répété chez les jeunes, parfois même en fin de grossesse ? Comment œuvrer à la désinstitutionnalisation de personnes atteintes de maladies mentales quand les familles qui les accueillent n'ont pas été préparées à ce nouveau choix de société ? En quoi les choix de société que nous faisons sont-ils porteurs de sens pour celles et ceux qui attendent de cette dernière qu'elle offre une clé de reconnaissance à la dignité humaine, à la justice, à l'équité ?

Ce foisonnement de problématiques à caractère éthique n'est pas sans atteindre le monde du travail, en l'occurrence les milieux professionnels. Au cœur des pratiques professionnelles en question, une crise secoue les occupations accréditées tout comme celles de l'ensemble de la société. Un groupe de chercheurs québécois ${ }^{1}$ affirme qu'au cœur de la quête d'identité professionnelle actuellement en développement, un professionnalisme serait à rechercher en dehors de la seule référence sociologique à la professionnalisation et à la profession comme telle. Les auteurs parlent d'un professionnalisme sans profession. En effet, n'est pas si loin de nous cette époque où un professionnel pouvait déclarer qu'il s'identifiait à son milieu de travail sur la base d'un sentiment d'appartenance à un réseau social. Porté par un sentiment collectif de participer avec d'autres à l'élaboration du projet de société, il pouvait naturellement compter sur différentes formes de normativité toutes aussi crédi- bles les unes que les autres et ce, tout simplement parce que ces dernières s'imposaient d'emblée.

Dans un tel contexte, le type de rapports que les professionnels avaient tendance à développer avec l'éthique renvoyait davantage à la construction d'outils de régulation propres à régir la vie professionnelle sous forme de codes, de guides de conduites, etc. L'approche privilégiée en était plus souvent une d'ordre hétérorégulatoire et sans autres contributions plus explicites des personnes concernées par ces modes d'encadrement. Qu'il se soit agi de construire des codes d'éthique, de déontologie, des guides d'éthique et de déontologie professionnelle ou simplement des listes de conseils pour réussir en affaires, on bâtissait, et on a encore tendance aujourd'hui à bâtir en investissant les énergies nécessaires pour s'assurer, sur un plan structurel le plus souvent, que la seule sécurité de l'organisation ne sera pas menacée. D'autres préoccupations et des questions surgissent. Comment protéger, dans un contexte syndical, un groupe de professionnels de baisses de salaires injustifiées ? Comment contrer les problématiques de violence en milieu de travail? Dans de tels contextes, les dirigeants d'institutions, d'entreprises, créent, sur la base de recommandations adressées par des subalternes, des politiques de régie interne. Ces formes de balises prennent rapidement place dans la législation et font partie de règlements à ne pas enfreindre sous peine de sanctions. Toutes aussi nécessaires les unes que les autres, ces mesures procurent-elles pour autant les ressources individuelles et collectives propres au développement de l'autonomie, de la prise en charge? 
Poser la question revient à dire que la référence à cette forme de sécurité sociale s'est transformée. « En l'absence de discours public commun sur l'identité professionnelle, il est impossible aujourd'hui de construire son lieu d'appartenance de manière significative. Le discours sur sa pratique ou sur son organisation, sur son lieu d'appartenance est nécessaire pour construire un "nous » à partir des « je » qui s'engagent volontairement à le créer ${ }^{2}$. Qu'en est-il alors de cette initiative que les professionnels sont appelés à prendre pour contribuer de façon dynamique et créatrice au déploiement du professionnalisme ou de l'idéal professionnel dont est porteuse l'organisation ou l'institution à laquelle ils appartiennent? Par le biais de quelle approche est-il possible de passer, pour donner lieu de façon motivante à cette forme de participation de la part des professionnels, à cette assomption sociale dans une perspective éthique ? Par quel moyen des professionnels pourraient-ils passer pour que celles et ceux qui désirent participer au changement puissent être et demeurer motivés à poursuivre leur engagement professionnel en ce sens? Voilà trois questions auxquelles nous aimerions apporter des éléments de réponse ici. En bout de ligne, nous aimerions avoir indiqué un chemin possible de prise en charge parmi d'autres de la part de professionnels à partir d'une approche éthique d'ordre autorégulatoire :

- de la participation à l'appropriation : une co-construction des bases d'un nouveau professionnalisme;

- l'approche éthique d'ordre autorégulatoire : vers une prise en charge individuelle et collective;

- d'une assomption individuelle et collective en service de garde : l'allégorie comme mode pédagogique d'intervention à l'articulation d'un professionnalisme.

\section{De la participation à l'appropriation : une co-construction des bases d'un nouveau professionnalisme}

Des bases pour une nouvelle construction sont en train de s'installer pour la co-construction d'un nouveau professionnalisme. Dans le prolongement de l'économie de service et des nouvelles professions qui sont apparues, un professionnalisme émerge sans pour autant que ce dernier soit automatiquement associé à une profession clairement déterminée. Ce changement social donne lieu à un déplacement important en matière d'éthique professionnelle. Qui dit éthique professionnelle dans pareil contexte renvoie à un idéal professionnel qui est en train de devenir vrai pour toute occupation soucieuse de s'inscrire dans l'idéal de ladite économie de service. La façon de servir l'autre sur le plan professionnel s'en trouve atteinte. Tout autant que le principal lieu d'émergence du sens, celui de la personne est remis en question. La relation à soi devient à ce point importante que, dans le monde des affaires, la motivation au travail constitue le centre des préoccupations. Comment développer une motivation au travail, un sentiment d'appartenance et un souci de contribuer au développement de l'organisation qui l'engage quand l'éthique personnelle et l'éthique professionnelle du travailleur arrivent mal à s'harmoniser en lui ? Pour être plus constructif, comment faire en sorte que la motivation, d'externe à l'individu, devienne une composante interne du professionnalisme auquel il aspire?

\section{Des bases pour une nouvelle construction sont en train de s'installer pour la co-construction d'un nouveau professionnalisme.}

Une première condition est l'engagement du sujet dans pareille aventure et sa motivation à s'en investir. Le professionnel s'engage avec l'objectif de participer de façon active et créatrice au changement du milieu professionnel auquel il se sent appartenir. On s'éloigne alors du modèle professionnel dépendant du milieu social déjà connu. Du professionnel cherchant à se modeler sur les règles pré-établies pour construire son intervention, ce dernier devient, avec d'autres, coconstructeur d'une démarche de vie professionnelle davantage empreinte de sens. Du lieu où chacun se trouve et dans un esprit de coopération, professionnels et clients s'unissent comme actrices et acteurs de l'articulation d'un projet de société de manière à conjuguer une expertise professionnelle dont les projets de vie respectifs des personnes constituent un moteur d'articulation de cette expertise.

L'éthique continue alors de renvoyer à la recherche de sens, tant sur les plans individuel et collectif que social et structurel. La démarche de réflexion à laquel- 
le elle renvoie force son interlocuteur, individu et collectivité, à rechercher à la frontière de la légitimité et de la légalité la décision la plus créative et responsable dans le but d'atteindre la plus grande cohérence possible dans les circonstances ${ }^{3}$. L'éthique professionnelle comme façon de se ressaisir des différentes formes de normativité, de l'action et des enjeux en présence desquels elle place les interlocuteurs constitue alors chaque jour pour soi, l'autre et autrui une occasion potentielle de changement. Entre alors en jeu la capacité de chacune et chacun de se responsabiliser de telle démarche. En effet, une équipe de travail capable de mettre clairement les mots, le contexte de ce qui constitue l'environnement éthique de la vie professionnelle qui la façonne au quotidien en vient graduellement à participer de l'intérieur aux perspectives qu'elle envisage pour les personnes et le groupe qu'elle forme. Comment illustrer concrètement une façon dont il est devenu possible pour une équipe interdisciplinaire du domaine de la santé de s'actualiser dans leur travail à travers une démarche éthique d'ordre autorégulatoire ? En voici un exemple.

\section{L'approche éthique d'ordre autorégulatoire : vers une prise en charge individuelle et collective}

Une infirmière avait un jour décidé de s'engager personnellement dans une démarche de compréhension éthique plus approfondie des problèmes de jugement professionnel auxquels elle faisait face en matière d'avortement répété de la part des jeunes femmes qui venaient la consulter dans l'institution de santé à laquelle elle appartenait. Soucieuse de continuer à faire équipe avec d'autres infirmières, médecins et travailleuses sociales qui, avec elle, étaient aux prises avec des dilemmes éthiques tels qu'encourager ou pas une jeune femme de seize ans à vivre un avortement après plus de trente semaines de grossesse, par exemple, elle choisit de les réunir.

Après leur avoir raconté comment elle en était arrivée à élucider le malaise qui l'habitait et les avenues de sens qui lui étaient apparues, elle assista à une initiative dont elle fut la première surprise. Le groupe décida de se constituer en comité régional d'éthique sur la question. L'approche qu'ils décidèrent d'emprunter a été celle utilisée par la collègue qui les avait invitées, soit une approche éthique d'ordre autorégulatoire.
Dans un premier temps, le seul fait d'accepter de se ressaisir individuellement et collectivement des faits auxquels elles étaient confrontées a demandé une grande énergie. Très rapidement sont apparues des prises de conscience importantes auxquelles elles faisaient face. Si, d'emblée, elles croyaient extérieurement qu'un des malaises qui les habitaient était dû aux opinions différentes qu'elles avaient sur le sujet, elles constatèrent par l'intérieur que leur adhésion à des valeurs différentes était devenue un lieu important d'interprétation des difficultés auxquelles elles faisaient face à travers leur pratique. L'une disait qu'elle ne voyait pas de problèmes à accompagner quelqu'une en l'amenant à faire le choix qu'elle désirait faire en matière d'avortement tout en l'appuyant en ce sens. Une autre cherchait régulièrement à s'assurer qu'elle avait bien présenté les prescriptions, conseils et recommandations à utiliser pour que la jeune femme décide en fonction de l'une d'elles. Une troisième intervenante disait que son souci était de bien présenter les risques encourus devant une interruption volontaire de grossesse (IVG), les différentes formes de normativités qu'elle risquait de négliger mais, qu'en bout de ligne, ce qui lui importait était que le choix d'une jeune femme ou d'un couple, quand il arrivait que les deux partenaires se présentent à l'entrevue, était que les personnes soient aussi à l'aise que possible dans les circonstances. De ce qu'elles croyaient être une divergence d'opinions en matière d'IVG, les professionnelles se rendirent vite compte en intériorisant le processus que leur malaise reposait sur une échelle de valeurs différente et différemment structurée. Cette prise de conscience a vite donné lieu à d'autres éléments de réflexion. De multiples conflits de valeurs apparurent.

Les valeurs personnelles qu'elles portaient pouvaient devenir des occasions de divergences importantes quant aux prises de position qu'elles étaient appelées à prendre. Ces valeurs pouvaient donner lieu à d'autres conflits de valeurs. Comment s'orienter dans une décision collective à prendre quand valeurs personnelles, valeurs professionnelles et valeurs organisationnelles semblent pousser une équipe de travail vers des choix divergents, parfois même opposés ? C'est alors que s'engagea de leur part un travail systématique de réflexion. Ils choisirent également de vivre les séquences d'une démarche éthique avec un superviseur de façon à s'approprier certains éléments de contenus. Mais ce que l'équipe recherchait surtout était une habilitation individuelle et collective à re- 
conduire progressivement de mieux en mieux une démarche éthique à son terme dans l'autonomie, la liberté et le respect de chacun et ce, de façon responsable

L'approche dialogique a constitué une première voie d'accès à mettre en place. À travers cette approche, il ne s'agit pas tellement d'associer des règles aux problématiques identifiées dans le but de dénouer les impasses, cette approche consiste plutôt à mobiliser les actrices et acteurs à rechercher ensemble dans et par l'action le meilleur choix à faire dans les circonstances. "L'idéal de la formation à l'autonomie responsable pourrait se résumer ainsi : développer la capacité de prendre des décisions dans lesquelles il existe le moins de dissonance possible entre le soi actuel et son héritage passé et le soi actuel et ses rapports aux autres en privé comme en public. » ${ }^{4}$

Une telle démarche éthique est un exercice de réflexion à caractère dialogique plus ou moins étendu dans le temps qui, dans un milieu professionnel, renvoie des actrices et acteurs à leur capacités internes à se ressaisir individuellement et collectivement d'une problématique comportant un ou des enjeux éthiques de manière à rendre désirables par d'autres leur capacité respective à se responsabiliser de celles qui leur incombent.

\section{Travailler à une prise en charge individuelle et collective d'une problématique de manière à se motiver soi et à en motiver d'autres à agir dans le même sens implique donc un engagement de la part du sujet.}

Une telle appropriation appelle une motivation à œuvrer en ce sens. Par motivation, nous entendrons ici «cette force qui stimule le comportement, dirige les conduites et nourrit la tendance à persévérer ${ }^{5}$. Pour que se poursuive le dialogue et que d'autres développent eux-mêmes de l'intérieur une motivation à agir en ce sens, des moyens pédagogiques ont été mis en place.

De quelle façon l'allégorie peut-elle devenir un moyen pédagogique privilégié pour que s'actualise chez le sujet professionnel une approche éthique à caractère autorégulatoire?

\section{D'une assomption individuelle et collective en service de garde : l'allégorie comme mode pédagogique d'intervention à l'articulation d'un professionnalisme}

Travailler à une prise en charge individuelle et collective d'une problématique de manière à se motiver soi et à en motiver d'autres à agir dans le même sens implique donc un engagement de la part du sujet. Cet engagement peut s'actualiser de différentes façons. Pour des services de garde du Québec, l'engagement a pris forme dans la mise sur pied d'un comité provincial d'éthique professionnelle. C'est en effet à la suite de quelques rencontres de sensibilisation à une gestion éthique du secret professionnel et de la confidentialité dans quelques régions du Québec que l'idée a germé, chez quelques intervenantes du milieu professionnel en question, de mettre en place un tel comité. Sa mission est de reconnaître et de promouvoir l'éthique professionnelle dans le réseau des centres de la petite enfance (CPE) et en service de garde au Québec. Deux objectifs donnent lieu à une mise en forme de cette mission: soutenir l'émergence de la dynamique éthique en service de garde et accompagner les $\mathrm{CPE}$ et les régions dans une démarche provinciale en éthique professionnelle. Les moyens privilégiés pour concrétiser la démarche ont donné lieu à la mise en place d'un site Web. La formation offerte ouvre sur la construction d'outils d'intervention de manière à ce que des actrices et acteurs se mobilisent individuellement et collectivement à œuvrer dans l'autonomie et de façon autorégulatoire à l'articulation d'une telle démarche éthique.

Une expérience en service de garde vécue en ce sens a donné lieu à un exercice fort prometteur de créativité. Dans un premier temps, un exercice semblable à celui réalisé par l'équipe interdisciplinaire d'une institution de santé fut mis en place par une équipe d'intervenantes et d'intervenants en service de garde. La prise de conscience s'est concrétisée lorsque des intervenantes et intervenants en service de garde ont réalisé le rôle stratégique qu'ils étaient appelés à exercer au sein de la famille québécoise. Certaines tâches routinières étaient devenues l'apanage de leurs us et coutumes. $\mathrm{Au}$ cours d'un processus de réflexion, l'accueil d'un enfant à son arrivée le matin est rapidement devenu un lieu potentiel enrichissant de responsabilisation pour la relation à soi, à l'autre, à autrui. La relation à soi pouvait se trouver enrichie par le biais d'une con- 
fidence transmise par un parent au sujet de son enfant, à savoir le danger potentiel d'avoir été victime de violence physique de la part de son père lors de sa visite chez ce dernier la veille. Dès lors, l'intervenante pouvait être mise en situation de saisir encore mieux la divergence existant entre ses valeurs personnelles et celles du parent en question: pour le parent, une question de sécurité, pour l'éducatrice, une question de professionnalisme. La relation à l'autre était nourrie par les incidences parfois nombreuses que cette courte démarche d'accueil pouvait susciter au sein de toute l'équipe d'intervenantes au cours de la journée : quoi dire, à qui, comment le dire ? La relation à autrui s'en trouvait influencée en raison de toutes les expertises susceptibles d'être réunies de façon prochaine ou lointaine pour que la situation en arrive à trouver le dénouement le plus heureux possible dans les circonstances (interventions spécialisées de psychologues, intervenants sociaux, etc.).

Au cours de l'exercice, l'idée vint de rechercher des moyens pédagogiques pour rendre encore plus facilement accessible à des interlocuteurs le changement qui se produit quand une situation est décrite, analysée et ressaisie par des agents de réflexion dans une perspective éthique. S'il est vrai que l'actualisation d'une démarche éthique peut faire passer les personnes impliquées dans une situation problématique d'un constat d'iniquité, d'injustice ou de manque de reconnaissance de la dignité à une action donnant lieu à une plus grande équité, une plus grande justice ou une dignité plus certaine, par quels moyens est-il possible de passer pour construire le professionnalisme au sein d'une organisation ou d'une institution sans que le seul moyen préconisé soit un code, un guide ou une liste de conseils, de sanctions, etc. ?

Celui que la conseillère d'un centre de la petite enfance (CPE) a utilisé est l'allégorie. « Nous la définirons comme une histoire réelle (anecdote) ou fictive dont le but est d'informer, d'éduquer, de guérir et de faire grandir. L'objectif de l'allégorie est d'attirer l'attention consciente de l'individu et de déjouer ses mécanismes de défense afin de lui permettre d'entrer en contact avec les forces de son inconscient, riche de possibilités et de solutions. ${ }^{6}{ }^{6}$ Tantôt, c'est la partie logique de la personne qui est rejointe. Mais à d'autres moments, l'affectif est davantage sollicité.

La conseillère pédagogique est donc partie d'une situation insatisfaisante vécue au sein du CPE auquel elle appartenait. Elle a d'abord procédé à une description du problème.

Sophie est éducatrice au centre de la petite enfance «Les petits mulots». Elle croit profondément que le professionnalisme d'une intervenante se mesure au degré d'autorité et de contrôle sur son groupe d'enfants. Au quotidien, ses interventions reflètent cette croyance. Elle agit de façon directive en attendant une réaction rapide de la part d'un enfant. Elle qualifiera ensuite cette réaction de comportement non acceptable. Elle hausse ensuite le ton et s'énerve régulièrement, ce qui a pour effet de surexciter les enfants du groupe. Les jeux de pouvoir prennent la place sur le plaisir partagé. Les enfants pensent à autre chose qu'à jouer. Le stress de l'éducatrice augmente en même temps que son insatisfaction. Le doute s'installe en elle. Elle en vient à vérifier auprès de l'éducatrice qui la remplace pendant sa journée de congé hebdomadaire si celle-ci vit la même dynamique au sein du groupe d'enfants. De quelle manière Sophie pourraitelle s'y prendre pour emprunter un comportement plus harmonieux?

En analysant la situation, la conseillère pédagogique en est arrivée à dénouer le dilemme en proposant que l'éducatrice change son mode d'intervention. Elle faisait cette proposition pour donner prépondérance aux valeurs d'harmonie, d'estime de soi et de plaisir. De là, quel outil d'intervention mettre à sa disposition ou à la disposition de l'éducatrice à qui Sophie s'était confiée pour dénouer l'impasse ? L'allégorie fut privilégiée comme moyen pédagogique pour dénouer l'impasse dans une perspective éthique d'ordre autorégulatoire. Voici cette allégorie ${ }^{7}$. Elle s'intitule «Les rennes du Père Noël » ${ }^{8}$.

Cette année, le Père Noël avait demandé à Sylvia pour la première fois, de rassembler les rennes de son traîneau. C'était une bien rude tâche pour une petite fille! Sylvia se sentait très fière, mais aussi très inquiète.

Sylvia savait qu'elle devait partir à la recherche des rennes sans plus tarder. Ils étaient quelque part dans la toundra vivant en liberté depuis Noël dernier. Ils ne se laisseraient sûrement pas attraper facilement et Sylvia devrait montrer beaucoup d'autorité.

Quand elle les vit jouant dans la neige, elle les reconnut tous: Fougère, Bruyère, Tempête, Lichen, Banquise, Roc, Éclair et Toundra. 
Sylvia pris sa grosse voix pour commander: «Allez! ...En avant! ...Par ici! Les rennes, surpris par ce ton grave, se retournèrent pour voir quel géant leur parlait sur ce ton. Mais ils obéirent et se dirigèrent vers la ferme. Seul Toundra faisait la mauvaise tête. Sylvia ne savait pas qu'il se considérait comme le chef et qu'il n'aimait pas les ordres.

Le lendemain, une grande nervosité régnait dans l'étable. Les rennes bondissaient et ruaient dans tous les sens. Sylvia leur donna de la nourriture et de l'eau. Elle fit la toilette de chaque renne. Elle voulait qu'ils aient un poil lisse et brillant pour les présenter au Père Noël. Elle les peigna et les brossa si fort et si longtemps que leurs oreilles commencèrent à rosir.

Sylvia fit sortir les rennes pour les atteler au trâेneau. Il fallait les placer deux par deux en rangée. Mais ils refusèrent de rester en ligne.

"Restez en ligne! Calmez-vous!", hurla Sylvia.

Mais les rennes galopaient en zigzag et elle eut bien du mal à les rattraper tous.

Le jour suivant, Sylvia réussit à atteler les rennes à l'intérieur de l'étable. Puis elle les fit sortir dehors pour leur apprendre à tourner à gauche et à droite en conservant leur place dans la rangée. Mais, tout alla de travers!

Les deux premiers rennes se bousculèrent par accident et tombèrent dans la neige en emmêlant leurs bois. À la vue des deux rennes couchés sur le dos, les quatre pattes en l'air, Sylvia cria aux autres rennes : "Stop! Stop! 》.

Les hurlements de Sylvia ne firent qu'exciter les autres rennes qui accoururent et trébuchèrent sur les rennes déjà au sol en s'accrochant les uns les autres. Ils avaient beau pousser et tirer de toutes leurs forces, ils ne pouvaient plus bouger.

"Oh non!", gémit Sylvia, " et c'est bientôt Noël!».

À la vue de tous les rennes immobilisés dans la neige, Sylvia fondit en larmes. Le vent du Nord passant par là lui murmura à l'oreille: "Lequel est le plus doux, un chuchotement ou la fourrure d'un renne?»

"C'est ma faute », dit-elle. "Je n'ai fait que crier au lieu de vous aider. Pardonnez-moi!"»

Et elle fit à chacun un gros bisou sur leur museau. "Demain, je m'y prendrai autrement: plus de hurlements, plus d'énervements. Et maintenant, je vais essayer de vous libérer. "
Au son nouveau de cette voix douce, les yeux des rennes s'illuminèrent et ils retrouvèrent le sourire. Au fur et à mesure qu'ils se réjouissaient, leurs bois se secouaient et se dénouaient. Et sans que Sylvia ne fit rien que partager leur gaieté, les rennes se retrouvèrent libres.

Sylvia raccompagna les rennes à l'étable. Elle lissa leur fourrure, brossa délicatement leurs oreilles et peigna leur crinière. Toundra lui caressa la joue.

Le lendemain, les rennes se mirent d'eux-mêmes en ligne, deux par deux, Toundra en tête avec Bruyère. Ils tournèrent à droite, à gauche quand Sylvia le leur demandait de sa voix douce. Ils s'exercèrent ainsi toute la journée.

Le soir venu, ils entendirent le tintement des grelots qui les appelait. C'était le Père Noël, prêt à partir, à côté de son traîneau rempli de cadeaux.

Sylvia conduisit les rennes en bon ordre jusqu'au traîneau. Le Père Noël lui sourit et la remercia de son beau travail. Et c'est ainsi que le magnifique attelage s'envola dans la nuit étoilée.

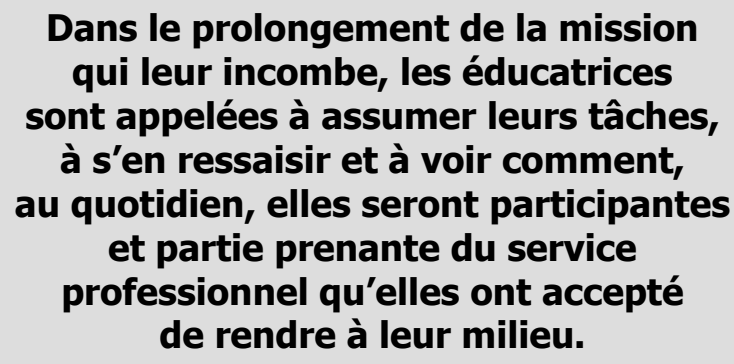

Le potentiel d'un tel moyen pédagogique pour présenter à Sophie des moyens de modifier son comportement avec les enfants était certes délicat. La conseillère pédagogique et le $\mathrm{CPE}$ se trouvaient aussi en présence d'un problème d'éthique professionnelle. D'abord, le CPE, par les personnes qu'il embauche, se doit d'agir avec professionnalisme dans le respect des différences dont sont porteurs les enfants et les familles qu'il accueille. Dans le prolongement de la mission qui leur incombe, les éducatrices sont appelées à assumer leurs tâches, à s'en ressaisir et à voir comment, au quotidien, elles seront participantes et partie prenante du service professionnel qu'elles ont accepté de rendre à leur milieu. En ce sens, un code d'éthique pourrait servir d'intermédiaire entre Sophie et le CPE pour indiquer qu'un changement serait souhaitable, voire souhaité, dans tel contexte. Les nombreuses étu- 
des prouvent toutefois qu'un tel instrument de régulation ne saurait suffire à provoquer de l'intérieur les changements attendus.

Un code s'adresse à la partie logique de l'être humain. Michel Dufour propose que le fait de solliciter le seul cerveau gauche a plutôt tendance à laisser l'interlocuteur dans la certitude qu'il a bien reçu l'information, mais que la transformation intérieure ne s'est pour autant pas réalisée. Interdire par exemple à un enfant de deux ans de jouer dans les panneaux d'armoire en l'absence de sa mère peut facilement lui donner l'idée de s'en amuser sans que l'interdiction de s'y rendre ait été intégrée pour autant. En sollicitant le cerveau droit, un message inconscient lui parvient. Lui proposer plutôt une image lui indiquant l'idée de génie qu'a eue la fourmi d'aller aider son amie à construire une petite maison pour abriter d'autres amis peut susciter chez lui, ne serait-ce que de façon inconsciente, que lui aussi aurait une idée de génie à en faire autant. Au lieu de lui interdire le jeu dans les panneaux d'armoire, la mère devient alors pour l'enfant une source de motivation à aller jouer ailleurs.

\section{Conclusion}

Les moyens de régulation mis en place au cœur de la vie en société dans une perspective éthique d'ordre hétérorégulatoire n'ont pas manqué depuis qu'il est davantage question de se rapprocher des comportements attendus à l'intérieur des organisations sur le plan professionnel, en l'occurrence. Si de tels moyens ont donné lieu à un encadrement mieux défini et plus circonscrit pour que s'engagent les changements souhaités, d'autres seraient aussi souhaités et espérés pour que s'opèrent de l'intérieur les modifications comportementales chez les individus et collectivités. Pour qu'une telle mobilisation se concrétise, un appel à la créativité est lancé.

L'initiative ici proposée de mettre de l'avant des moyens pédagogiques d'intervention tels que l'allégorie se veut de ceux-là. Le Laboratoire de recherche et d'intervention en éthique professionnelle de l'Université du Québec à Chicoutimi a commencé à offrir à ses intervenants d'intégrer à leur coffret d'intervention un tel outil. Des démarches d'accompagnement à une plus grande valorisation de l'estime de soi de mê- me que les apprentissages de base à une gestion éthique de la communication sont également annoncés. De la part des milieux qui logent au même laboratoire, une demande d'accompagnement s'ouvrent alors sur les perspectives de mise en œuvre de la créativité des intervenants pour que se constitue toute une banque d'outils d'intervention en vue d'un développement concerté d'éthique professionnelle. L'objectif visé est qu'un plus grand effet structurant, tel qu'attendu de la part de ces organisations, puisse produire comme fruit l'émergence d'une approche éthique d'ordre autorégulatoire, tant au plan individuel que collectif chez ceux qui sont appelés à participer au déploiement de la mission de ladite organisation dans une perspective de plus grande cohérence interne et d'une participation encore plus certaine à l'articulation de la cohésion sociale.

\section{Notes et références}

1 Voir Legault, Georges, dir. (2003). Crise d'identité professionnelle et professionnalisme, Sainte-Foy Presses de l’Université du Québec.

2 Desaulniers, Marie-Paule et al. (2003). « Le professionnalisme: vers un renouvellement de l'identité professionnelle », dans G.A. Legault, dir. Crise d'identité professionnelle et professionnalisme, Sainte-Foy, PUQ, p. 197.

3 Lamoureux, Henri (2002). «Responsabilités professionnelles et dilemmes éthiques : l'exigence de la cohérence », Intervention, $\mathrm{n}^{\circ} 117, \mathrm{p} .17$.

4 Legault, Georges A.(1999). Professionnalisme et délibération éthique, Sainte-Foy, PUQ, p. 89.

5 Papalia, Diane E. (1988). Introduction à la psychologie, Montréal, McGraw-Hill, p. 320.

6 Dufour, Michel (1993). Allégories pour guérir et grandir, recueil d'histoires métaphoriques, Chicoutimi, Les Éditions JCL, p. 24.

7 Il s'agit d'un outil d'intervention construit par Madame Denise Lepage conseillère pédagogique. Cet outil est une adaptation d'un conte écrit et illustré par Jan Bret et édité chez Gautier-Languereau.

8 Cette allégorie a été construite à partir du livre de conte Les rennes du Père Noël de Jan Bret, aux Éditions GautierLanguereau. 


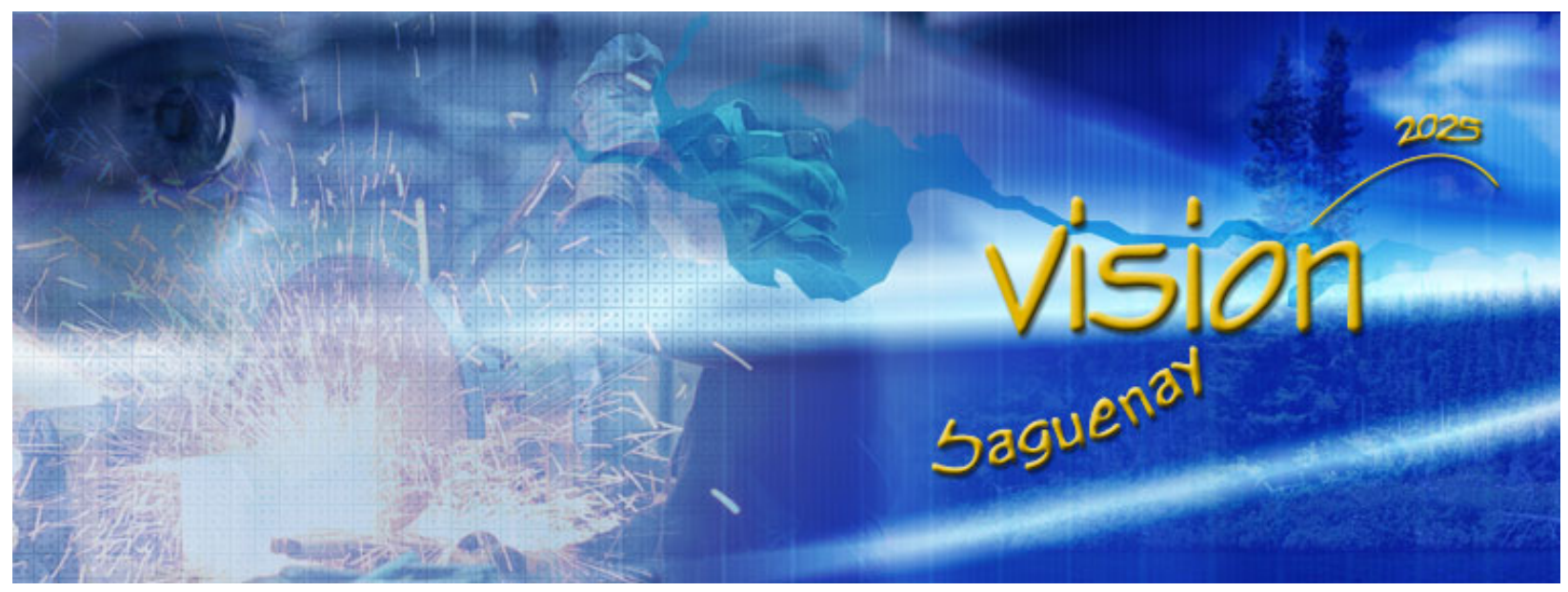

Vision Saguenay 2025 est une initiative du Centre de recherche sur le développement territorial (CRDT) de l'UQAC, ayant trois finalités principales:

- stimuler la réflexion collective de nature prospective;

- faire circuler l'information tacite;

- soutenir l'apprentissage collectif à saisir les enjeux communautaires.

Le projet de prospective territoriale de Vision Saguenay 2025 s'articule autour de quatre axes :

- les éléments généraux de Vision 2025;

- la consolidation territoriale de la Cité;

- l'intégration de la Cité dans sa périphérie immédiate et éloignée;

- le repositionnement de la Cité et de sa vaste région sur l'échiquier national, continental et mondial.

Activités tenues par Vision Saguenay 2025 :

\section{2 mars 2003}

Séminaire : « L'armature urbaine »

11 septembre 2003

Séminaire : « Le rôle de la culture dans le développement d'une capitale régionale »

10 octobre 2003

Séminaire : « Les jeunes, leur place et leur avenir à Saguenay »

4 novembre 2003

Séminaire : «L'enjeu du tertiaire moteur »

\section{8 novembre 2003}

Séminaire : « L'enjeu de l'agroalimentaire pour la capitale

Saguenay "

1er décembre 2003

Séminaire : «L'enjeu de l'entrepreneuriat dans la Cité

saguenéenne en maturation économique »

\section{2 décembre 2003}

Séminaire : "La démocratie »

Séminaire : « La gouvernance »

\section{9 janvier 2004}

Lancement du Portail de Vision Saguenay 2025

\section{2 janvier 2004}

Séminaire : « Le développement social : apanage du mouvement communautaire ou projet de collectivité ?»

\section{6 février 2004}

Séminaire: "L'enjeu de la forêt »

\section{3 février 2004}

Séminaire : «L'aménagement du territoire à Ville de Saguenay » 20 février 2004

Séminaire : « Le rôle des médias dans le développement local et régional $»$

\section{7 février 2004}

Séminaire : "L'économie des collectivités autochtones »

\section{9 mars 2004}

Séminaire : « L'enjeu de l'entrepreneurship coopératif »

7 et 8 avril 2004

Colloque « Le Devenir de Saguenay »

\section{7 mai 2004}

Séminaire : « L'internationalisation dans les PME de

l'aluminium»

3 juin 2004

Séminaire : « Comment l'éducation peut-elle contribuer à forger l'identité individuelle et collective?»

10 septembre 2004

Forum sur les défis d'avenir de Ville de Saguenay et de sa vaste région

\section{8 octobre 2004}

Séminaire : « L'enjeu de l'hydroélectricité au Saguenay-LacSaint-Jean »

Vision Saguenay 2025 : 555, boul. de l'Université, Saguenay (Québec) G7H 2B1

Téléphone : (418) 545-5011, poste 4037 Télécopieur : (418) 545-5012

Courriel : vsag2025@uqac.ca Web :www.uqac.ca/vsag2025 